\title{
Mapping the effects of drugs on the immune system
}

\author{
Brian A Kidd ${ }^{1,2,7}$, Aleksandra Wroblewska ${ }^{1,7}$, Mary R Boland ${ }^{3}$, Judith Agudo ${ }^{1}$, Miriam Merad ${ }^{4-6}$, \\ Nicholas P Tatonetti ${ }^{3}$, Brian D Brown ${ }^{1,5,6} \&$ Joel T Dudley ${ }^{1,2}$
}

\begin{abstract}
Understanding how drugs affect the immune system has consequences for treating disease and minimizing unwanted side effects. Here we present an integrative computational approach for predicting interactions between drugs and immune cells in a system-wide manner. The approach matches gene sets between transcriptional signatures to determine their similarity. We apply the method to model the interactions between 1,309 drugs and 221 immune cell types and predict 69,995 interactions. The resulting immune-cell pharmacology map is used to predict how five drugs influence four immune cell types in humans and mice. To validate the predictions, we analyzed patient records and examined cell population changes from in vivo experiments. Our method offers a tool for screening thousands of interactions to identify relationships between drugs and the immune system.
\end{abstract}

Pharmaceutical drugs of all types and classes influence the immune system ${ }^{1-4}$ but the mechanisms of these perturbations are often poorly understood. Some drugs target immune cells specifically to treat immunological diseases, such as B-cell lymphomas (e.g., rituximab (Rituxan $)^{5}$ ), whereas others have broad immunosuppressive or antiinflammatory effects (e.g., thalidomide (Thalomid) ${ }^{6}$, leflunomide $(\text { Arava })^{7}$ or sirolimus (Rapamune $)^{8}$ ). However, many drugs that were not developed to be immunomodulatory are nevertheless associated with mild to severe immune reactions. For example, several anti-infectives, anti-convulsants and anti-diabetic drugs are believed to induce the skin hypersensitivity reaction urticaria ${ }^{9-11}$, and psychoanaleptics, such as respiridone (Risperdal), memantine (Namenda) and citalopram (Celexa), have the rare, but life-threatening side effect of an immune-complex hypersensitivity called Stevens-Johnson syndrome ${ }^{1}$. Our lack of understanding of the global interactions between pharmaceuticals and the immune system confounds drug development, conceals potentially serious side effects of marketed

${ }^{1}$ Department of Genetics and Genomic Sciences, Icahn School of Medicine at Mount Sinai, New York, New York, USA. ${ }^{2}$ Icahn Institute for Genomics and Multiscale Biology, Icahn School of Medicine at Mount Sinai, New York, New York, USA. ${ }^{3}$ Department of Biomedical Informatics, Systems Biology and Medicine, Columbia University Medical Center, New York, New York, USA. ${ }^{4}$ Department of Oncological Sciences, Icahn School of Medicine at Mount Sinai, New York, New York, USA. ${ }^{5}$ Tisch Cancer Institute, Icahn School of Medicine at Mount Sinai, New York, New York, USA. ${ }^{6}$ Immunology Institute, Icahn School of Medicine at Mount Sinai, New York, New York, USA. ${ }^{7}$ These authors contributed equally to the work. Correspondence should be addressed to J.T.D. (joel.dudley@mssm.edu) or B.D.B. (brian.brown@mssm.edu).

Received 7 February; accepted 11 August; published online 30 November 2015; doi: $10.1038 /$ nbt.3367 compounds ${ }^{12-14}$ and limits discovery of drugs that could be repurposed for immune diseases.

Published studies on the effects of drugs on immune cells have mainly examined the consequences of administering one drug to a single cell type ${ }^{15,16}$. Even when high-throughput screens have been performed, they usually have focused on a specific target or readout (e.g., changes in select cell surface markers) ${ }^{17-19}$ and ignored other perturbations to the system. In the present report, we build on previous systems-level approaches that compare and integrate differential expression profiles of disease with drug perturbation profiles to discover potential new drug indications ${ }^{20-22}$. Recent large-scale collaborative efforts have produced compendia of molecular profiles for both pharmaceutical drugs ${ }^{23}$ and immune cells ${ }^{24}$. To our knowledge, systematic integration and analysis of chemogenomic and immunogenomic data have not been performed.

We integrated drug perturbation data obtained with human cancer cells and gene expression data obtained from mouse immune cells. Our analysis quantifies the likelihood that a drug affects an immune cell state change in the form of an 'immunemod score'. In total, we generated 304 immune cell state transitions from 221 immune cell types. We studied all combinations of 1,309 drugs and 304 immune cell state transitions, and found 69,995 significant interactions (of 397,936 possible interactions). From these interactions, we constructed an immune-cell pharmacology (IP) map of predicted drug-immune cell connections, which includes both known interactions as well as ones that have-to our knowledge-not been reported previously. To address concerns about integrating data across species, we examined predictions in both mouse and humans. We performed in vivo experimental validation of three predictions and obtained $100 \%$ agreement. In addition, we found evidence in patient data that supported our predicted interactions between drugs and immune cells in two independent sets of electronic medical records. Our results suggest that integrative computational analysis can improve understanding of the effects of drugs on the immune system and provide a framework for rational manipulation of these effects.

\section{RESULTS}

\section{Generating molecular signatures of immune cells}

The Immunological Genome Project (ImmGen) is the largest publicly available compendium of genome-wide transcriptional expression profiles for more than 250 distinct immunological cell states in mice ${ }^{25-27}$. The data comprise 14 categories of immune cell types collected from 25 tissue locations (Supplementary Fig. 1). These states reflect diverse stages of lineage differentiation, collected from various tissues, using an assortment of genetic variants, in response to stimulations with chemicals, bacteria or viruses, and at separate effector stages. One challenge with using ImmGen data for probing 
immune perturbations is that gene expression profiles were captured at a single state, which provides limited information on cellular response to external stimuli. Thus we created a data set that reflects immune cell responses to perturbations by generating differentially expressed gene signatures between two immunological states that differ by a single parameter (e.g., cell types with identical surface markers isolated from separate tissues or two cell types that differ by one surface marker such as naive vs. memory $\mathrm{CD} 4^{+} \mathrm{T}$ cells).

We compiled a set of 304 immune cell state change signatures from 221 unique cell types in the ImmGen compendium to explore how drug perturbations alter the immune system (Fig. 1a,c and Supplementary Table 1). The full ranked lists are provided in Supplementary Table 2. These signatures group by similar cell types when clustered by the Jaccard distance between sets of the extreme fold-change genes (Supplementary Fig. 2). The average Jaccard distances between related cell types exceeded the overall average background distance (Supplementary Fig. 3) and showed significant differences between immune cell subsets $\left(P=6 \times 10^{-15}\right.$, ANOVA).

\section{Generating drug chemogenomic profiles}

The Connectivity Map (CMap) is a data repository of genome-wide transcriptional expression profiles collected from 6,100 experimental conditions of 1,309 unique compounds applied to human cell lines ${ }^{23}$. Each perturbation is represented by a list of differentially expressed genes that we ranked based on fold change. To capture the consensus profile of a compound across conditions, we merged multiple experiments (i.e., different drug concentrations or cell lines) for the same compound into a single prototype ranked list (PRL), using a hierarchical majority-voting scheme $\mathrm{e}^{28,29}$ (Fig. 1a). The collection of PRLs created a comprehensive resource for developing a systematic screening tool to look for connections between drug perturbations and immunological states (Supplementary Table 3 ).

\section{IP map construction}

We created a system-wide interaction map between drugs and immune cells by matching the 1,309 drug perturbation profiles in CMap to the 304 immune cell state changes we curated from the ImmGen compendia. Our matching algorithm evaluates the similarity between two transcriptional expression patterns by comparing the top- and bottomranked genes from both profiles ${ }^{20,21}$ (Fig. 1b, Supplementary Fig. 4 and Online Methods). Specifically, we tested the similarity between the immunological state-change profiles (state B vs. state A) to each of the drug perturbation profiles (treated vs. untreated) by computing an immunemod score based on the overlap of the top- and bottom-ranked genes in each profile. A positive immunemod score indicates that the specific drug treatment

Figure 1 Constructing the drug-cell interactome. (a) Schematic overview of the data integration and processing steps. (b) Schematic depiction of the matching algorithm and score distribution. (c) Summary of immunological cell state changes studied. (d) Diagram of the influence of drugs on immune cell state changes predicted by the sign of the immunemod score.

\section{a}

b

profile is similar to immune cell state $B$ and suggests the drug biases the immune cell toward state $B$, whereas a negative score signals the drug treatment shifts the cell toward state A (Fig. 1b,d).

To evaluate the significance of our predicted drug-cell interactions, we generated random drug perturbation profiles for each compound and repeated the analysis 1,000 times for each immune cell state change (Supplementary Fig. 4a). A complete computational integration of the CMap and ImmGen data sets produced 397,936 potential connections between drugs and immune cell state changes. To assess whether a predicted connection was robust, we varied the set size of the top- and bottom-ranked genes used for the matching algorithm and recalculated all $\sim 400,000$ immunemod score $P$-value pairs. The proportion each drug-cell interaction was significant among all gene set sizes provided a relative weight for each predicted interaction (Supplementary Fig. 4b,c). Larger weights indicate that a given drugcell interaction depends less on the set size chosen to calculate the immunemod score and signifies a robust connection. This selection process enabled discovery of previously unknown interactions while minimizing spurious connections (Supplementary Fig. 5).

Using the significant and robust interactions, we made connections between drugs and immune cell state changes to generate a comprehensive IP map. The IP map contains 69,995 connections (Supplementary Table 4) that are significant at a false-discovery rate (FDR) $<5 \%$ and that appear in $>85 \%$ of gene set sizes. Although every drug showed a significant association with at least one of the 304 immunological state changes, the most frequent number of state transitions is 26 , and 144 drugs influence 100 or more state changes (Supplementary Fig. 6). Drugs predicted to influence the largest number of immune cell state transitions include potent immunomodulators, many of which induce significant immunosuppression (Table 1). Drugs with immunomodulatory activity (e.g., anti-inflammatory agents, anti-histamines and immunosuppresants) show a significant enrichment for immune cell interactions $(E=1.5, P=0.002, E=1.4, P=0.04, E=2.1, P=0.02$, respectively, and Supplementary Table 5).

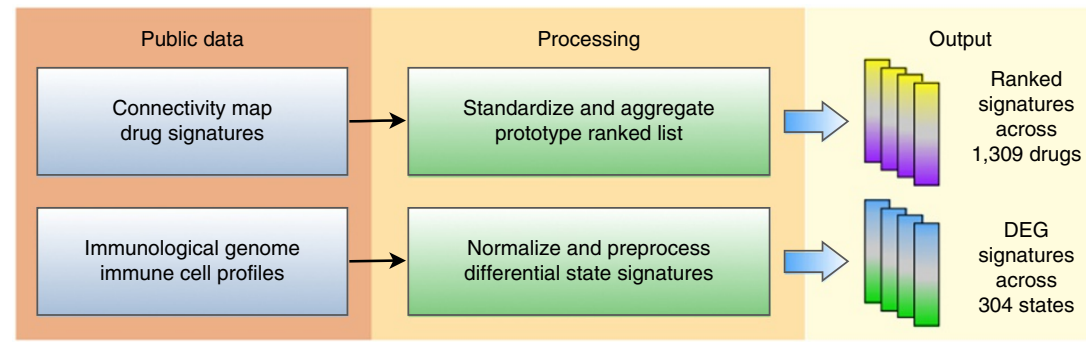

Calculate overlap in extremes of

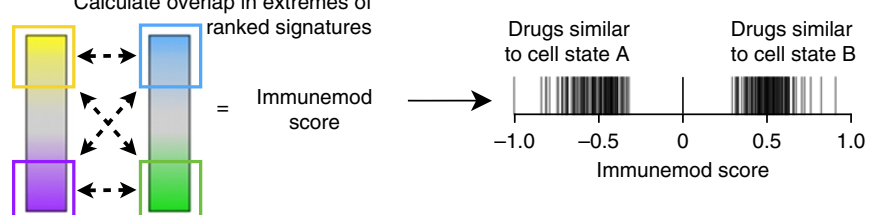

C

\begin{tabular}{lccr}
\hline Category & A & B & Number \\
\hline Subset & Naive & Memory & 143 \\
Tissue & BM & Spleen & 80 \\
Genetic & WT & KO & 33 \\
Time & Early & Late & 32 \\
Perturbation & Unstim & Stim & 15 \\
Disease & Arthritis & Normal & 1 \\
\hline
\end{tabular}

d

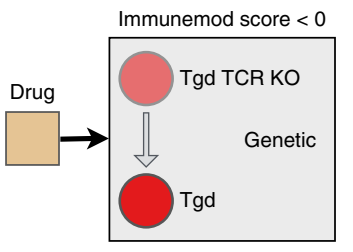

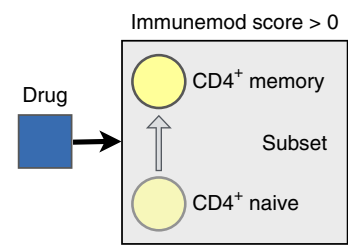


Table 1 Drugs predicted to influence largest number of immune state changes

\begin{tabular}{lcll}
\hline Drug & State changes & \multicolumn{1}{c}{ Status } & \multicolumn{1}{c}{ Drug class } \\
\hline Puromycin & 138 & Experimental & Aminonucleoside antibiotic \\
Quinostatin & 129 & Experimental & PI3K inhibitor \\
Deptropine & 129 & Approved & Anti-histamine \\
Gliclazide & 127 & Approved & Anti-diabetic \\
Fluspirilene & 127 & Approved & Anti-psychotic \\
Irinotecan & 127 & Approved & Topoisomerase inhibitor \\
Pyrvinium & 126 & Experimental & Anti-helmintic \\
Bepridil & 126 & Approved & Calcium channel blocker \\
Daunorubicin & 126 & Approved & Anthracycline \\
Celastrol & 125 & Experimental & Anti-inflammatory \\
Niclosamide & 125 & Approved & Anti-helmintic \\
Pimozide & 124 & Approved & Anti-psychotic \\
\hline
\end{tabular}

\section{Global properties of the IP map}

To examine the global landscape of the IP map, we used the immunemod score as a similarity metric and organized the complete set of drug and immune cell interactions through unsupervised hierarchical clustering (Fig. 2a). We found that drugs with similar therapeutic classes cluster together. For example, anti-psychotics (clozapine (Clozaril), loxapine (Adasuve), haloperidol (Haldol) and fluphenazine) formed a cluster, as did purine analogs (mercaptopurine (Purixan) and tioguanine), and calcium channel blockers (dexverapamil, bepridil and perhexiline). These three clusters are predicted to interact with the largest number of immune cell subset transitions. Drug clusters also showed enrichment for the same molecular target. For example, the anti-diabetic drugs troglitazone (Rezulin) and rosiglitazone (Avandia) both target PPARG and ACSL4 as part of their mechanism of action for reducing blood glucose. Based on their immunemod scores, these drugs are predicted to influence T cell and natural killer (NK) cell differentiation, providing a potential explanation for the therapeutic benefits observed in patients with autoimmune disease ${ }^{30,31}$.

We identified 28 drugs associated with later stages of lineage development across multiple cell types (e.g., stem/progenitor and pre-B cells; Fig. 2a and Supplementary Table 6). These drugs include

a

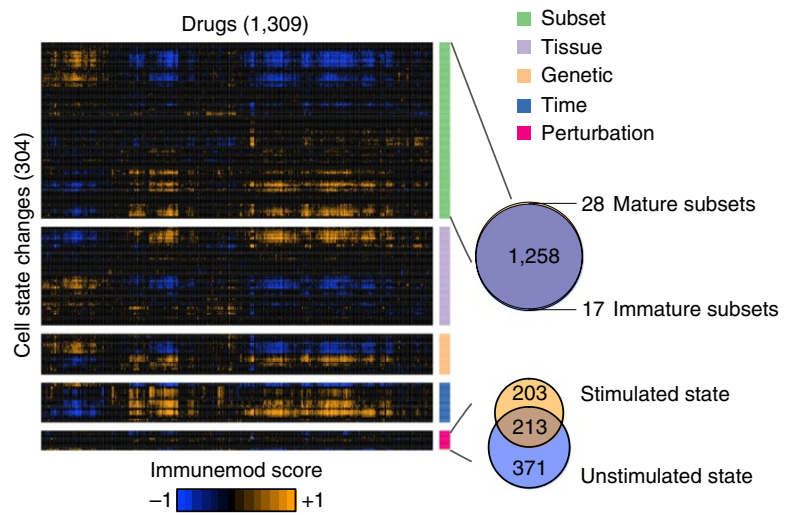

b

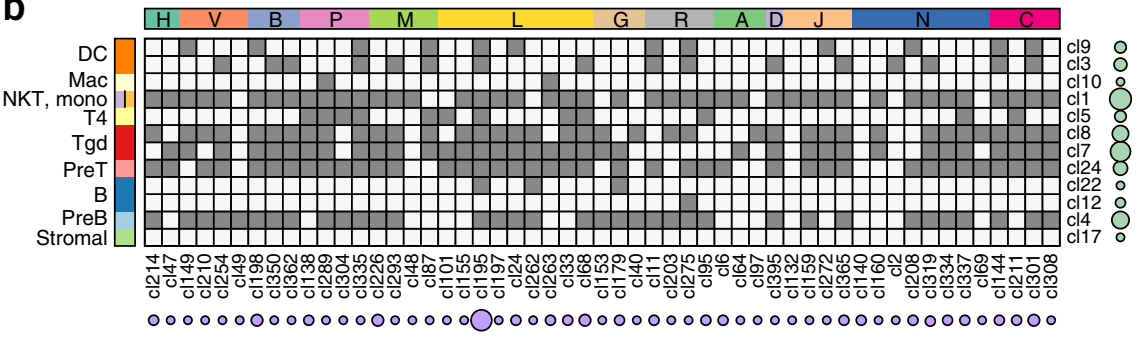

compounds used to treat diseases of metabolism and the nervous, musculo-skeletal and respiratory systems, or are anti-infectives. Moreover, these 28 drugs are enriched for (i) agents with immunosuppressant, anti-psoriatic and dopaminergic activity, and (ii) compounds that target the chromatin-associated enzyme PARP1, which is a key regulatory molecule for differentiation and proliferation ${ }^{32,33}$. By contrast, 17 compounds influence immature hematopoietic cell subsets (Fig. 2a and Supplementary Table 7). These compounds aren't associated with a single therapeutic class, yet their molecular targets are enriched for processes associated with oxidoreductase activity and alkylation repair, both of which are important for differentiation and maintaining stem cell integrity ${ }^{34,35}$.

To further characterize the drug-immune cell interactome, we performed unsupervised hierarchical clustering with multiscale bootstrap resampling ${ }^{36}$. Out of the 143 cell type changes, 119 fit into one of 25 stable cell clusters $(P<0.05$, multiscale bootstrap analysis) (Supplementary Table 8). Almost half (13/25) of the stable cell clusters exhibited a significant over-enrichment $(E>2, P<0.05)$ for one or more cell types (Supplementary Table 9). By comparison, 1,089 drugs out of the 1,309 in total fell into one of 409 stable drug clusters $(P<0.05$, multiscale bootstrap analysis) (Supplementary Table 10). Almost 87\% (356/409) of the stable drug clusters showed a significant over-enrichment $(E>4, P<0.05)$ for one or more therapeutic classes (anatomical therapeutic chemical (ATC) classification levels 1-3) or a molecular target (Supplementary Table 11). For example, 88 drug clusters showed significant enrichment for at least one ATC classification system level 1 description. The enrichments are driven by an abundance of anti-thrombotic agents or vitamin $\mathrm{K}$ and other hemostatics (B), contrast agents or diagnostic radiopharmaceuticals (V), and alkylating agents, cytotoxic antibiotics, hormone antagonists or immunosuppressants (L) (Supplementary Fig. 7).

To examine the features of stable clusters in greater detail, we identified 53 drug clusters enriched for a therapeutic class and molecular

Figure 2 Overview of the IP map. (a) Clustered heat map of the drugs and immune cell state changes organized by the immunemod score. Venn diagrams reflect the number of drugs that shift immune cell states in a specific or nonspecific direction. (b) Two-dimensional heat map of the stable cell and drug clusters with a significant enrichment for at least one cell type (rows) or a level 1 therapeutic class (columns). Cell types include: DC, dendritic cell; Mac, macrophage; NKT, natural killer T cell; mono, monocyte; T4, CD4 ${ }^{+} \mathrm{T}$ cell; Tgd, gamma-delta T cell; PreT, pre T cell; B, B cell; PreB, pre-B cell; Stromal, stromal cell. Therapeutic classes include: $\mathrm{H}$, systemic hormonal preparations, excluding sex hormones and insulins; $V$, various; $\mathrm{B}$, blood and blood-forming organs; $P$, antiparasitic products; $M$, musculoskeletal system; $L$, anti-neoplastic and immunomodulating agents; G, genito-urinary system and sex hormones; $R$, respiratory system; $A$, alimentary tract and metabolism; D, dermatologicals; J, anti-infectives for systemic use; $\mathrm{S}$, sensory organs; $\mathrm{N}$, nervous system; and C, cardiovascular system. Areas of corresponding circles represent the number of cells or drugs per cluster (2-14 cells, 2-13 drugs). Cluster labels (e.g., cl214) refers to the cluster number in Supplementary Tables 9 and 10 for cells and drugs, respectively. Gray squares indicate predicted interaction between at least one cell-drug pair in the pairs of clusters. 
Figure 3 Predicting immune cell influence from the IP map. (a) Circular layout of the predicted connections between drugs and cell types (all connections statistically significant $P<0.05$ with FDR $<5 \%$ ). Line widths correspond to the number of interactions. The diagram has been organized by sorting the cell types counter-clockwise (drugs clockwise) in order of decreasing number of connections. Single-letter codes for each drug follows the anatomical therapeutic classification system as described in Figure 2. (b) Subnetwork showing the drug hubs and islands (square nodes in the center and periphery, respectively) and their predicted interactions with immune cell subsets (circles). Each square node represents a single drug (e.g., the four center nodes labeled $L$ represent, counter clockwise from top, the drugs daunorubicin, azacitidine, vorinostat and methotrexate). (c) Drugs identified in the IP map based on their immunemod score and predicted to increase neutrophil and monocyte frequencies in the blood. (d) Validation of drug prediction in immune cells from humans. Drug influence (+, drug; -, no drug) on measured cell counts from laboratory values of patients in the Mount Sinai Electronic Medical Records (MSSM EMR, cell counts and frequencies). Bar heights represent median count (neutrophil) or frequency (monocyte) values and error bars reflect the 25th and 75th percentiles). Significance assessed via Wilcoxon rank sum test.

target, and intersected these clusters with the 13 cell clusters enriched for one or more cell type (Fig. 2b). The intersecting clusters revealed that specific immune cell subsets (e.g., pre-lymphocytes, monocytes, NKT cells, and gamma-delta T cells) overlap with multiple drug categories, whereas other subsets (e.g., B cells and macrophages) intersect with a couple categories. A few drug clusters (e.g., cl195, cl335) influenced multiple cell types, whereas other clusters (e.g., cl2, cl48, cl49, cl97) influenced a single cell type. The drug cluster with the greatest overlap across immune cells (cl195) was enriched for antineoplastic drugs that are cytotoxic antibiotics (e.g., doxorubicin (Doxil) and mitoxantrone).

We discovered a strong positive association between the number of molecular targets for a given drug and the number of interactions predicted to influence immunological state transitions $\left(P=1.7 \times 10^{-5}\right.$, linear regression). When we examined adverse drug interactions using the side effect resource (version 2$)^{1}$, we found no relationship between the reported number of side effects for a drug and the number of immune cell interactions ( $P=0.8$, linear regression). However, as side effect data have a broad frequency distribution and are difficult to measure accurately ${ }^{2}$, this lack of correlation may reflect the variation inherent in the bias of capturing and reporting side effects.

\section{Connections and substructures in the IP map}

To examine possible immunological outcomes that might result from connections in the IP map, we focused on immune cell state changes

Table 2 Drugs predicted to influence largest number of immune subset changes

\begin{tabular}{lcll}
\hline Drug & $\begin{array}{c}\text { Subset } \\
\text { changes }\end{array}$ & Status & \multicolumn{1}{c}{ Drug class } \\
\hline Irinotecan & 60 & Approved & Topoisomerase inhibitor \\
Puromycin & 58 & Experimental & Aminonucleoside antibiotic \\
Deptropine & 55 & Approved & Anti-histamine \\
Tyrphostin AG-825 & 54 & Experimental & Tyrosine kinase inhibitor \\
O175029-0000 & 54 & Experimental & Unknown \\
Daunorubicin & 54 & Approved & Anthracycline \\
Medrysone & 53 & Approved & Corticosteroid \\
Bepridil & 52 & Approved & Calcium channel blocker \\
Etacrynic acid & 52 & Approved & Loop diuretic \\
Alsterpaullone & 52 & Experimental & Cyclin-dependent kinase inhibitor \\
Primaquine & 51 & Approved & Aminoquinoline \\
Procaine & 50 & Approved & Local anesthetic \\
Gliclazide & 50 & Approved & Anti-diabetic \\
Cinchonine & 50 & Experimental & Alkaloid \\
Piperidolate & 50 & Approved & Anti-cholinergic \\
\hline
\end{tabular}

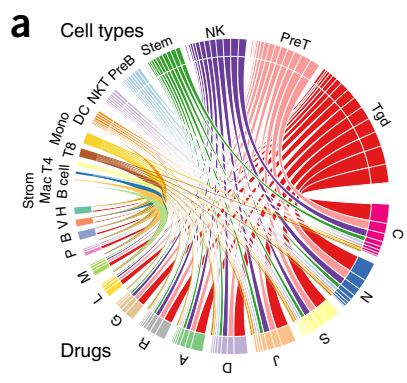

C

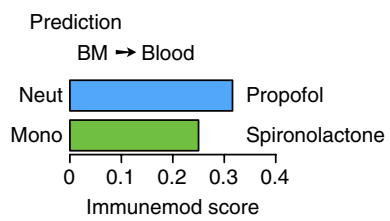

b

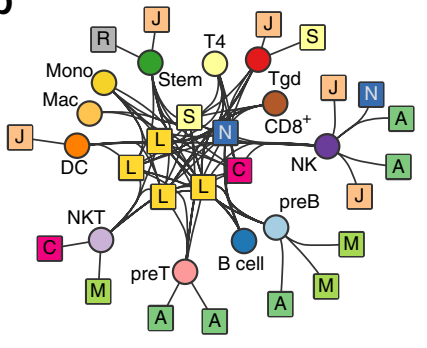

d

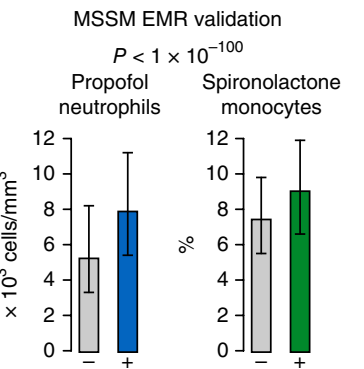

between cell subsets or tissues. Drugs and cell state transitions were organized by therapeutic class and category of immune cell state transition (e.g., within a subset or between tissue) to provide an overview of all the predicted connections that are statistically significant (Fig. 3a and Supplementary Fig. 8). We identified promiscuous drugs by their interactions with a large number of subset transitions (Table 2). Given the uneven cell type distribution within the subset state changes (Supplementary Table 1), we defined drug hubs based on interactions with the greatest number of cell types, which we hypothesize could have the largest influence on the immune system. By contrast, drug islands were defined on the basis of interacting with the fewest number of cells (Fig. 3b).

Hubs were enriched for anti-neoplastic drugs $(E=13.8, P<$ $1 \times 10^{-5}$; Supplementary Fig. 9), which could be expected given the influence these compounds have on immune cells ${ }^{1,2}$. Hubs were also enriched for nervous system compounds, such as the selective serotonin reuptake inhibitor zimeldine $(E=1.3, P<0.04)$, which was pulled off the market due to a rare, but severe adverse reaction leading to the autoimmune condition known as Guillain-Barré syndrome ${ }^{37}$, and the anti-seizure drug topiramate (Topamax), which was shown to be an effective treatment for inflammatory bowel disease in a preclinical model ${ }^{21}$ (Supplementary Fig. 10a). By contrast, drug islands were enriched for metabolic drugs that included the antidiabetic compounds gliquidone and metformin (Fortamet, Glumetza; Supplementary Fig. 10b). This metabolic island in the IP map mirrors the low connectivity found in metabolic diseases in the human disease network ${ }^{38}$.

To explore drugs predicted to influence a portion of the adaptive immune system, we identified a subnetwork based on the largest magnitude immunemod scores for T-cell subsets and tissues (Supplementary Fig. 11). This subnetwork includes more than 1,000 compounds predicted to influence $\mathrm{CD} 4^{+}$or $\mathrm{CD}^{+}$subsets, with 113 and 202 compounds unique to each group, respectively. The top immunemod score for this subnetwork is between $\mathrm{CD}^{+}{ }^{+} \mathrm{FoxP} 3^{+}$ $\mathrm{T}$ cells and guanfacine (Tenex, Intuniv; Supplementary Figs. 12 and 13a). Guanfacine is an $\alpha 2 \mathrm{~A}$ receptor (ADRA2A) agonist used for lowering blood pressure and treating attention deficit hyperactivity disorder (ADHD) ${ }^{39}$.

To verify that the immunemod score identifies a drug's influence on a specific cell subset, we administered the anti-hypertensive drug 
a

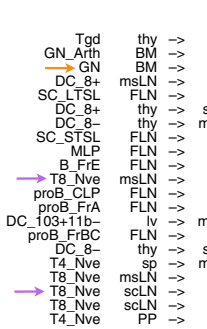

b

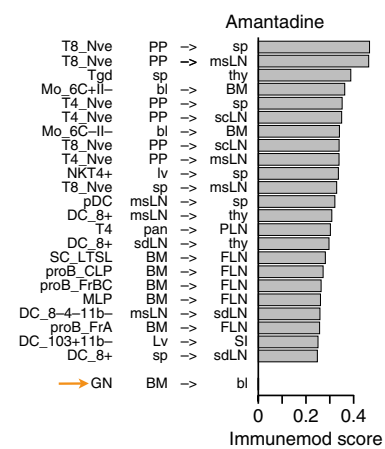

C

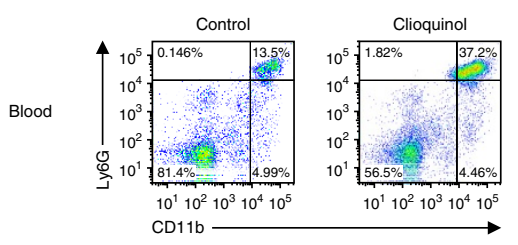

$$
\begin{aligned}
& \text { Bone } \\
& \text { marrow }
\end{aligned}
$$

$$
\stackrel{5}{3}
$$

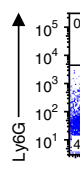

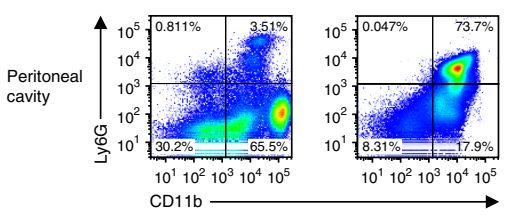

d
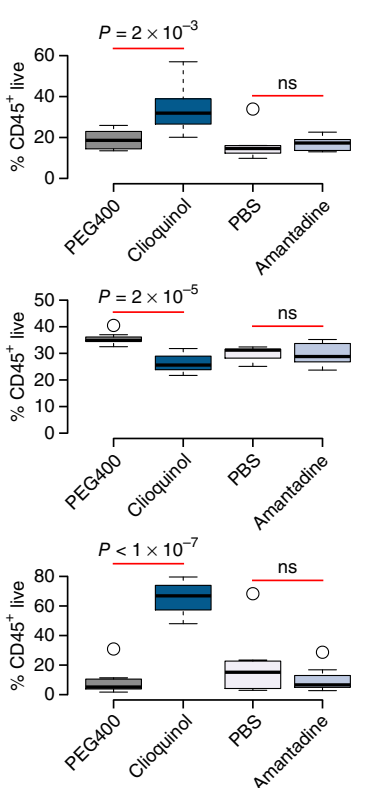

e mod score
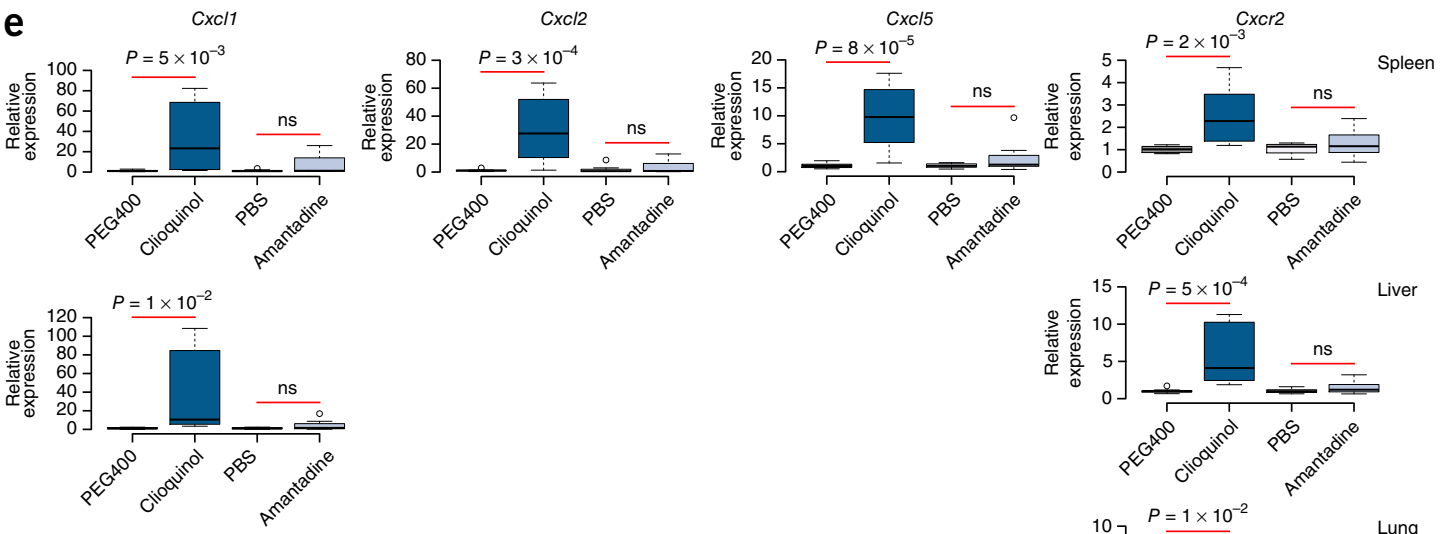

\section{f}
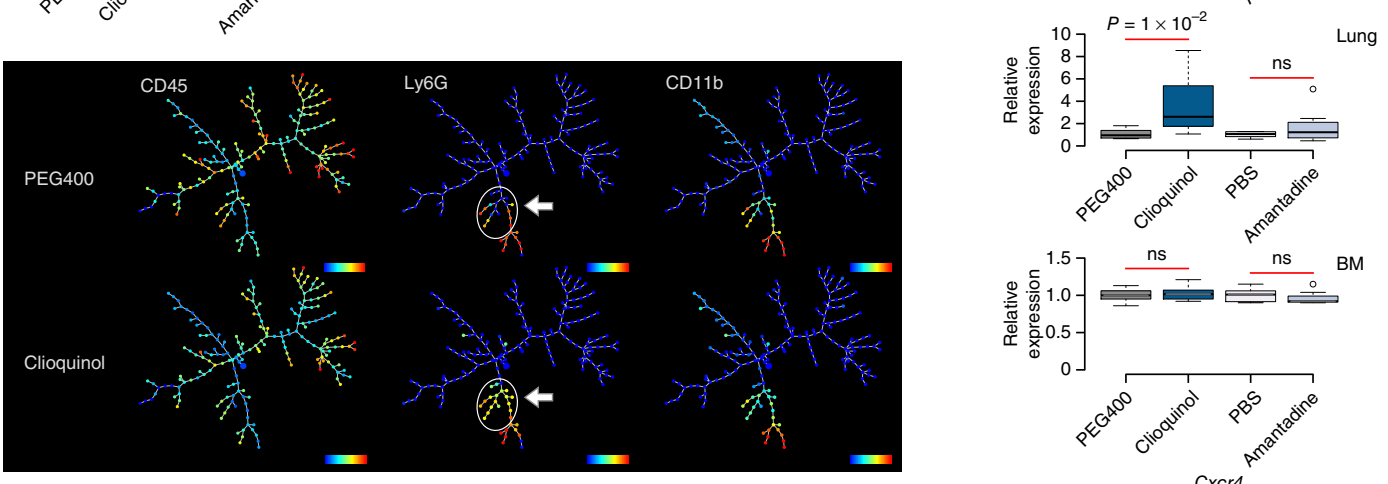

Figure 4 Influencing immune cell migration using the IP map. (a,b) Immunemod scores for influencing immune cells between tissues using clioquinol (a) or amantadine (b). Labels indicate the direction of influence for the drug to a cell type between tissues $x \rightarrow y$, and bar width is the magnitude of the immunemod score. Shown are the significant and robust drug-cell interactions. Orange arrows reflect predicted top interaction with clioquinol and non-interaction with amantadine. Purple arrows point to predicted interactions with additional experimental validation. (c) Biaxial flow cytometry plots of $\mathrm{Ly}_{6 \mathrm{G}}{ }^{+} \mathrm{CD} 11 \mathrm{~b}^{+}$cells collected from various tissues of treated and untreated mice. Cells were gated on $\mathrm{CD} 45^{+} \mathrm{DAPI}-$ population. (d) Frequencies of $\mathrm{Ly}_{6 \mathrm{G}}{ }^{+} \mathrm{CD} 11 \mathrm{~b}^{+}$neutrophils collected from blood, bone marrow and the peritoneal cavity ( $n=8$ per group). Black bars reflect the median expression of all samples in each condition and the bottom and top of the boxes represent the 25th and 75th percentiles, respectively. Whiskers on boxplots represent the max/min values up to $1.5 \times$ the interquartile range (IQR). Data exceeding 1.5× IQR shown as open circles. Data are representative of three to four experiments. Significance assessed by ANOVA. (e) Quantitative analysis of neutrophil marker gene expression. The abundance of each transcript in spleen, liver, lung and bone marrow (BM) was calculated relative to the appropriate control (PEG400 for clioquinol or PBS for amantadine). Each experimental condition includes eight samples. Significance assessed by ANOVA. (f) SPADE trees of CyTOF data collected from cells in spleen. Arrows highlight Ly6G+CD $11 \mathrm{~b}^{+}$population increase after clioquinol treatment. Horizontal color scales represent median intensity for marker indicated (blue, low; red, high). 
guanfacine to mice and measured the percentages of regulatory T-cell subsets isolated from spleens. Based on the immunemod score direction, we reasoned the frequency of regulatory $\mathrm{T}$ cells should increase following treatment with guanfacine. In comparison to untreated mice, the treated mice showed a $5 \%$ increase in the average frequency of $\mathrm{CD} 62 \mathrm{~L}^{+}$cells within the $\mathrm{CD} 4^{+} \mathrm{FoxP} 3^{+} \mathrm{T}$-cell compartment $(42.0 \%$ vs. $37.0 \%: n=15$ treated vs. $n=14$ untreated, $P=0.01$; Supplementary Fig. 13b).

When we examined the CD8 ${ }^{+}$subsets, the top immunemod score was to the anti-Parkinsonian drug trihexyphenidyl (Artane). One molecular target for trihexyphenidyl is the muscarinic acetylcholine receptor M1 (CHRM1). When the gene that encodes for this molecular target is knocked out in a mouse model, $\mathrm{CD} 8^{+}$cells from these mice exhibit defective cytotoxic capability ${ }^{40}$.

\section{Validation of drug-immune cell interactions in humans}

Immune cell data were collected from mice, and drug perturbation data were gathered from human cancer cell lines. One concern with integrating these data is whether the findings from mice translate to humans. We tested whether interactions predicted by the IP map could be observed in humans by examining immune cell counts of patients administered drugs versus untreated patients. To compare patient populations, we examined complete blood counts for more than 2.3 million electronic medical records in the Mount Sinai Hospital System and selected individuals who were treated and had blood cell counts collected within 1 month of receiving a drug.

Given the constraints of routine clinical laboratory tests found in electronic medical records, we restricted IP map predictions to two common drugs predicted to influence monocytes and neutrophils. The IP map predicted the general anesthetic propofol (Diprivan) and the anti-hypertensive spironolactone (Aldactone) would increase neutrophils and monocytes, respectively (Fig. 3c). Propofol increased neutrophil counts by 2,500 cells $/ \mathrm{mm}^{3}$, and spironolactone increased monocyte frequencies $1.6 \%$ (Fig. 3d). Although the cell population changes were small, both shifts were significant $\left(P<1 \times 10^{-100}\right.$, Wilcoxon rank sum). Furthermore, the neutrophil increase shifted most patients beyond the upper normal range. To validate these observations, we examined the same drug-immune cell pairings in the electronic medical records of Columbia University Medical Center. This independent data source showed the same direction and significance for both drugs and their predicted influence on immune cells (Supplementary Table 12).

\section{Validation of clioquinol influencing neutrophil migration}

To assess the accuracy and specificity of a predicted interaction in the IP map, we experimentally validated the influence of the drug clioquinol on neutrophil migration from the bone marrow to the blood. This hypothesis emerged from a prediction with a top immunemod score (Fig. 4a), as well as the desire to identify a drug that could modify immune system dynamics between tissues and would be straightforward to evaluate with an abundant cell type in vivo. Moreover, neutrophil regulation plays a critical role in health and disease so new drugs that modify their kinetics might have therapeutic potential ${ }^{41-43}$.

We selected two drugs for the experiment on the basis of their immunemod scores and $P$-values. These statistical metrics identified the highest immunemod score, and the corresponding lowest $P$-value, for the predicted pairing between neutrophils and clioquinol (Fig. 4a). Clioquinol is an anti-fungal and anti-protozoal drug without a known mechanism of action, but the compound has been tested in a preclinical model for Alzheimer's disease ${ }^{44,45}$. As a control, we selected the anti-viral and anti-Parkinsonian drug amantadine (Symmetrel) as a control drug because our algorithm predicted an immunemod score of zero for the amantadine-neutrophil interaction (Fig. 4b). Based on the immunemod scores, we reasoned that clioquinol would influence neutrophil migration from the bone marrow to the blood, whereas amantadine would have no influence on neutrophil migration (Fig. 4 b).

To evaluate the predicted influence of clioquinol on neutrophils in vivo, we injected C57BL/6 mice with clioquinol or vehicle control $(n=8$ treated and $n=8$ untreated). Following the treatment with clioquinol, but not the control, neutrophils were recruited to the peritoneal cavity (Fig. 4c,d and Supplementary Fig. 14). In mice treated with clioquinol, more than $70 \%$ of the hematopoietic cells in the cavity were neutrophils, whereas in mice treated with vehicle alone, less than $5 \%$ were neutrophils, similar to untreated mice. This recruitment coincided with neutrophil mobilization from bone marrow to blood (Fig. 4c,d and Supplementary Fig. 14). Moreover, qPCR analysis of collected tissue samples revealed increased abundance of transcripts of neutrophil-specific chemokines such as Cxcl1, Cxcl2 and Cxcl5 (Fig. 4e). We also detected Cxcr2 transcript, which suggests infiltration of neutrophils in the analyzed samples.

As predicted, in mice treated with amantadine there was no significant change in the frequencies of neutrophils in blood, bone marrow or peritoneal cavity (Fig. 4d). To survey a broader array of immune cell changes following treatment, we performed mass cytometry (CyTOF) using a panel of 19 markers to evaluate cell differences in the spleens of mice treated with clioquinol or vehicle control. Consistent with what we observed by flow cytometry, neutrophil numbers increased $\left(\right.$ Ly6G ${ }^{+} \mathrm{CD} 11 \mathrm{~b}^{+}$cells) after treatment with clioquinol (Fig. 4f). Furthermore, when we examined the complete set of markers using SPADE trees ${ }^{46}$, we found an increase in naive CD $8^{+}$cells $\left(\mathrm{CD} 45^{+}\right.$ $\mathrm{CD}^{+}{ }^{+} \mathrm{CD} 4^{-} \mathrm{CD} 8^{+} \mathrm{Thy}_{1.2}{ }^{+} \mathrm{TCRb}^{+} \mathrm{CD} 62 \mathrm{~L}^{\text {hi }} \mathrm{CD} 44^{\text {lo }}$ ) (Supplementary Fig. 15), which was also predicted by our algorithm albeit with a lower immunemod score (Fig. 4a).

\section{DISCUSSION}

We describe an integrative computational approach to map the effects of drugs on immune function. We compared chemogenomic and immunogenomic profiles and created an immunemod score to quantify the likely influence of a drug perturbation on an immune cell based on the overlap of their transcriptional profiles. Although the complete set of interactions between drugs and immune cells is larger than what we have modeled here, our systematic examination of almost 400,000 potential interactions is a step toward mapping this massive space. In vivo experiments to confirm one predictionthat the selective alpha-2A adrenergic receptor agonist guanfacine increases the proportion of regulatory T cells-suggested that a drug used to treat hypertension and anxiety might be repurposed to promote peripheral tolerance. To examine the utility of using the IP map to identify drugs with the highest immunemod scores for a given immunological state transition, we predicted that the change in neutrophil proportions between the blood and bone marrow would be most influenced by the drug clioquinol. This inference is supported by the proportions of neutrophils collected from blood and bone marrow and the pattern of neutrophil-specific genes expressed in various tissues. A previous study that used compounds to manipulate Cxcr 2 and Cxcr4 expression levels showed neutrophil mobilization patterns similar to what we observed ${ }^{47}$. Although clioquinol has neurotoxic effects, these findings imply its potential use as a short-term neutrophil booster in certain contexts. Additionally, this finding suggests that our approach could enable the discovery of compounds that control neutrophil kinetics to resolve inflammatory responses ${ }^{48}$. 
A limitation of the IP map is that it combines data sets from mouse and human, and therefore the predicted connections might not translate to human immune cells. Several recent studies have shown both similarities and differences between the transcriptional profiles of immune cells in mice and humans ${ }^{49-52}$. However, both of our predictions were confirmed in electronic medical records at Mount Sinai and in an independent data set from Columbia University. Taken together, our computational analyses and experimental results suggest that the IP map captures immune responses in both humans and mice.

Another limitation is that the CMap drug profiles were generated on a limited set of cancer cell lines using whole-genome transcriptional profiling. Although the efforts of the LINCS project (http://www. lincsproject.org/) will greatly expand the number of compounds and include more cell lines, it is likely worthwhile to generate immunespecific CMap signatures on at least a subset of immune cell types to further evaluate and improve the immunemod scoring method. Efforts such as the extensive transcriptional profiling of human cell lineage differentiation ${ }^{53}$ must be extended for a more comprehensive picture of human immunity, which will help us better understand how the ImmGen data will translate across species. We did not use chemical structure information, and we acknowledge that incorporating structural information and data other than transcriptional profiles ${ }^{54,55}$ would provide a more complete picture of the complexity of drug effects on the immune system.

The statistical bioinformatics method we used for systematically exploring drug-immune cell interactions follows a KolmogorovSmirnov (KS) approach similar to that used by numerous other studies $^{20,23,56}$. This method has been useful at identifying numerous biological connections that have been subsequently validated by experimentation. However, a limitation of the traditional KS approach using transcriptional data is an assumption of statistical independence among transcripts. Others have recently proposed potential solutions for this limitation ${ }^{57-60}$. When we incorporated a principal component analysis (PCA)-based approach ${ }^{57}$ into our methods, the $P$-values did rise as expected. Under this alternative null model, the number of significant interactions decreases by about a factor of 3 from the independent shuffling method (Supplementary Fig. 5). However, it appears that PCA-based correction may be overly pessimistic at low FDR thresholds ${ }^{57-60}$. This observation seems to be reflected in our own analysis where we find that all of the experimentally tested and validated interactions fall above the significance threshold subsequent to PCA-adjusted permutation. To our knowledge, there has not yet been a systematic analysis of the various proposed enrichment analysis methods on chemogenomic data based on uncorrelated gene sets. Systematic evaluation of permutation and expression de-correlation approaches for large-scale chemogenomic connectivity mapping is a fruitful area for future studies, especially as the chemogenomics community embraces reduced probe set arrays using the L1000 platform.

The apparently unknown interactions identified in the IP map may include many that warrant experimental follow-up. Other possible applications of our data include studying the contribution of immune cells to adverse drug reactions, the role of immune cell subsets in cancer and other diseases, and combination drug therapies. Moreover, global trends extracted from our data could provide guidelines and specific predictions on how to manipulate immune cells, uncover drug mechanisms of action, and select alternative compounds from the same therapeutic category with fewer immune cell side effects.

\section{METHODS}

Methods and any associated references are available in the online version of the paper.
Note: Any Supplementary Information and Source Data files are available in the online version of the paper.

\section{ACKNOWLEDGMENTS}

We thank G. Hoffman and B. Readhead for useful conversations about the computational methods and suggestions on the manuscript, L. Li for assistance with the electronic medical records from Mount Sinai, K. Oguntuyo for assistance with the $\mathrm{qPCR}$ reactions, and A. Rahman for sample preparation and acquisition on the CyTOF. This work was supported by the US National Institutes of Health grants R01AI104848, R33CA182377 and R01AI113221 (to B.D.B.), U54CA189201 and R01DK098242, and Jonathan E. Gray TCI Young Scientist Cancer Research Award (to J.T.D.).

\section{AUTHOR CONTRIBUTIONS}

B.A.K. and J.T.D. conceived of and designed the study. B.A.K. implemented the computational methods and performed the data analysis. A.W., J.A., M.M. and B.D.B. designed the experiments. A.W. conducted all in vivo assays. M.R.B. analyzed electronic medical records from Columbia University Medical Center B.A.K. and J.T.D. wrote the manuscript with review and feedback from A.W., B.D.B. and N.P.T.

\section{COMPETING FINANCIAL INTERESTS}

The authors declare competing financial interests: details are available in the online version of the paper.

Reprints and permissions information is available online at http://www.nature.com/ reprints/index.html.

1. Kuhn, M., Campillos, M., Letunic, I., Jensen, L.J. \& Bork, P. A side effect resource to capture phenotypic effects of drugs. Mol. Syst. Biol. 6, 343 (2010).

2. Tatonetti, N.P., Ye, P.P., Daneshjou, R. \& Altman, R.B. Data-driven prediction of drug effects and interactions. Sci. Transl. Med. 4, 125ra131 (2012).

3. Beghi, E. \& Shorvon, S. Antiepileptic drugs and the immune system. Epilepsia 52 (suppl. 3), 40-44 (2011).

4. Røge, R., Moller, B.K., Andersen, C.R., Correll, C.U. \& Nielsen, J. Immunomodulatory effects of clozapine and their clinical implications: what have we learned so far? Schizophr. Res. 140, 204-213 (2012).

5. Maloney, D.G. et al. IDEC-C2B8 (Rituximab) anti-CD20 monoclonal antibody therapy in patients with relapsed low-grade non-Hodgkin's lymphoma. Blood 90, 2188-2195 (1997).

6. Vogelsang, G.B. et al. Thalidomide for the treatment of chronic graft-versus-host disease. N. Engl. J. Med. 326, 1055-1058 (1992).

7. Fox, R.l. et al. Mechanism of action for leflunomide in rheumatoid arthritis Clin. Immunol. 93, 198-208 (1999).

8. Vézina, C., Kudelski, A. \& Sehgal, S.N. Rapamycin (AY-22,989), a new antifungal antibiotic. I. Taxonomy of the producing streptomycete and isolation of the active principle. J. Antibiot. (Tokyo) 28, 721-726 (1975).

9. Romano, A. \& Caubet, J.C. Antibiotic allergies in children and adults: from clinical symptoms to skin testing diagnosis. J. Allergy Clin. Immunol. Pract. 2, 3-12 (2014).

10. Kim, Y.J. et al. Cross-reactivity to acetaminophen and celecoxib according to the type of nonsteroidal anti-inflammatory drug hypersensitivity. Allergy Asthma Immunol. Res. 6, 156-162 (2014).

11. Sicherer, S.H. \& Leung, D.Y. Advances in allergic skin disease, anaphylaxis, and hypersensitivity reactions to foods, drugs, and insects in 2013. J. Allergy Clin. Immunol. 133, 324-334 (2014).

12. Alvir, J.M., Lieberman, J.A., Safferman, A.Z., Schwimmer, J.L. \& Schaaf, J.A. Clozapine-induced agranulocytosis. Incidence and risk factors in the United States. N. Engl. J. Med. 329, 162-167 (1993).

13. Ward, K.E., Archambault, R. \& Mersfelder, T.L. Severe adverse skin reactions to nonsteroidal antiinflammatory drugs: A review of the literature. Am. J. Health Syst. Pharm. 67, 206-213 (2010).

14. Garratty, G. Drug-induced immune hemolytic anemia. Hematology (Am Soc Hemato Educ Program) 2009, 73-79 (2009).

15. Cipolletta, D. et al. PPAR-gamma is a major driver of the accumulation and phenotype of adipose tissue Treg cells. Nature 486, 549-553 (2012).

16. Krönke, J. et al. Lenalidomide causes selective degradation of IKZF1 and IKZF3 in multiple myeloma cells. Science 343, 301-305 (2014).

17. Walker, R.G. et al. High throughput screens yield small molecule inhibitors of Leishmania CRK3:CYC6 cyclin-dependent kinase. PLoS Negl. Trop. Dis. 5, e1033 (2011).

18. Emert-Sedlak, L.A. et al. Effector kinase coupling enables high-throughput screens for direct HIV-1 Nef antagonists with antiretroviral activity. Chem. Biol. 20, 82-91 (2013).

19. Bellucci, R. et al. Tyrosine kinase pathways modulate tumor susceptibility to natural killer cells. J. Clin. Invest. 122, 2369-2383 (2012).

20. Sirota, M. et al. Discovery and preclinical validation of drug indications using compendia of public gene expression data. Sci. Transl. Med. 3, 96ra77 (2011).

21. Dudley, J.T. et al. Computational repositioning of the anticonvulsant topiramate for inflammatory bowel disease. Sci. Transl. Med. 3, 96ra76 (2011). 
22. Jahchan, N.S. et al. A drug repositioning approach identifies tricyclic antidepressants as inhibitors of small cell lung cancer and other neuroendocrine tumors. Cancer Discov. 3, 1364-1377 (2013).

23. Lamb, J. et al. The Connectivity Map: using gene-expression signatures to connect small molecules, genes, and disease. Science 313, 1929-1935 (2006).

24. Heng, T.S., Painter, M.W. \& Consortium, I.G. The Immunological Genome Project: networks of gene expression in immune cells. Nat. Immunol. 9, 1091-1094 (2008).

25. Jojic, V. et al. Identification of transcriptional regulators in the mouse immune system. Nat. Immunol. 14, 633-643 (2013).

26. Benoist, C., Lanier, L., Merad, M., Mathis, D. \& Immunological Genome, P. Consortium biology in immunology: the perspective from the Immunological Genome Project. Nat. Rev. Immunol. 12, 734-740 (2012).

27. Miller, J.C. et al. Deciphering the transcriptional network of the dendritic cell lineage. Nat. Immunol. 13, 888-899 (2012).

28. Iorio, F. et al. Discovery of drug mode of action and drug repositioning from transcriptional responses. Proc. Natl. Acad. Sci. USA 107, 14621-14626 (2010).

29. Iorio, F., Tagliaferri, R. \& di Bernardo, D. Identifying network of drug mode of action by gene expression profiling. J. Comput. Biol. 16, 241-251 (2009).

30. Zhang, X. \& Young, H.A. PPAR and immune system-what do we know? Int. Immunopharmacol. 2, 1029-1044 (2002).

31. Lewis, J.D. et al. Rosiglitazone for active ulcerative colitis: a randomized placebocontrolled trial. Gastroenterology 134, 688-695 (2008).

32. Rouleau, M., Patel, A., Hendzel, M.J., Kaufmann, S.H. \& Poirier, G.G. PARP inhibition: PARP1 and beyond. Nat. Rev. Cancer 10, 293-301 (2010).

33. Jagtap, P. \& Szabo, C. Poly(ADP-ribose) polymerase and the therapeutic effects of its inhibitors. Nat. Rev. Drug Discov. 4, 421-440 (2005).

34. Maugeri-Saccá, M., Bartucci, M. \& De Maria, R. DNA damage repair pathways in cancer stem cells. Mol. Cancer Ther. 11, 1627-1636 (2012).

35. Johannessen, T.C., Bjerkvig, R. \& Tysnes, B.B. DNA repair and cancer stem-like cells-potential partners in glioma drug resistance? Cancer Treat. Rev. 34, 558-567 (2008).

36. Suzuki, R. \& Shimodaira, H. Pvclust: an R package for assessing the uncertainty in hierarchical clustering. Bioinformatics 22, 1540-1542 (2006).

37. Fagius, J., Osterman, P.O., Siden, A. \& Wiholm, B.E. Guillain-Barre syndrome following zimeldine treatment. J. Neurol. Neurosurg. Psychiatry 48, 65-69 (1985).

38. Goh, K.-l. et al. The human disease network. Proc. Natl. Acad. Sci. USA 104, 8685-8690 (2007).

39. Kolar, D. et al. Treatment of adults with attention-deficit/hyperactivity disorder. Neuropsychiatr. Dis. Treat. 4, 389-403 (2008).

40. Fisahn, A. et al. Muscarinic induction of hippocampal gamma oscillations requires coupling of the M1 receptor to two mixed cation currents. Neuron 33, 615-624 (2002).

41. Summers, C. et al. Neutrophil kinetics in health and disease. Trends Immunol. $\mathbf{3 1}$, 318-324 (2010)

42. Fortunati, E., Kazemier, K.M., Grutters, J.C., Koenderman, L. \& Van den Bosch, v.J. Human neutrophils switch to an activated phenotype after homing to the lung irrespective of inflammatory disease. Clin. Exp. Immunol. 155, 559-566 (2009).
43. Beyrau, M., Bodkin, J.V. \& Nourshargh, S. Neutrophil heterogeneity in health and disease: a revitalized avenue in inflammation and immunity. Open Biol. 2, 120134 (2012).

44. Cherny, R.A. et al. Treatment with a copper-zinc chelator markedly and rapidly inhibits beta-amyloid accumulation in Alzheimer's disease transgenic mice. Neuron 30, 665-676 (2001).

45. Grossi, C. et al. Clioquinol decreases amyloid-beta burden and reduces working memory impairment in a transgenic mouse model of Alzheimer's disease. J. Alzheimers Dis. 17, 423-440 (2009).

46. Qiu, P. et al. Extracting a cellular hierarchy from high-dimensional cytometry data with SPADE. Nat. Biotechnol. 29, 886-891 (2011).

47. Devi, S. et al. Neutrophil mobilization via plerixafor-mediated CXCR4 inhibition arises from lung demargination and blockade of neutrophil homing to the bone marrow. J. Exp. Med. 210, 2321-2336 (2013).

48. Robertson, A.L. et al. A zebrafish compound screen reveals modulation of neutrophi reverse migration as an anti-inflammatory mechanism. Sci. Transl. Med. 6, 225ra229 (2014).

49. Seok, J. et al. Genomic responses in mouse models poorly mimic human inflammatory diseases. Proc. Natl. Acad. Sci. USA 110, 3507-3512 (2013).

50. Lin, S. et al. Comparison of the transcriptional landscapes between human and mouse tissues. Proc. Natl. Acad. Sci. USA 111, 17224-17229 (2014).

51. Cheng, Y. et al. Principles of regulatory information conservation between mouse and human. Nature 515, 371-375 (2014)

52. Shay, T. et al. Conservation and divergence in the transcriptional programs of the human and mouse immune systems. Proc. Natl. Acad. Sci. USA 110, 2946-2951 (2013).

53. Novershtern, N. et al. Densely interconnected transcriptional circuits control cell states in human hematopoiesis. Cell 144, 296-309 (2011)

54. Stepan, A.F. et al. Structural alert/reactive metabolite concept as applied in medicinal chemistry to mitigate the risk of idiosyncratic drug toxicity: a perspective based on the critical examination of trends in the top 200 drugs marketed in the United States. Chem. Res. Toxicol. 24, 1345-1410 (2011).

55. Evans, D.C. \& Baillie, T.A. Minimizing the potential for metabolic activation as an integral part of drug design. Curr. Opin. Drug Discov. Devel. 8, 44-50 (2005).

56. Subramanian, A. et al. Gene set enrichment analysis: a knowledge-based approach for interpreting genome-wide expression profiles. Proc. Natl. Acad. Sci. USA 102 15545-15550 (2005).

57. Nam, D. De-correlating expression in gene-set analysis. Bioinformatics 26 , i511-i516 (2010).

58. Wu, D. \& Smyth, G.K. Camera: a competitive gene set test accounting for inter-gene correlation. Nucleic Acids Res. 40, e133 (2012).

59. Yaari, G., Bolen, C.R., Thakar, J. \& Kleinstein, S.H. Quantitative set analysis for gene expression: a method to quantify gene set differential expression including gene-gene correlations. Nucleic Acids Res. 41, e170 (2013).

60. Rahmatallah, Y., Emmert-Streib, F. \& Glazko, G. Gene Sets Net Correlations Analysis (GSNCA): a multivariate differential coexpression test for gene sets. Bioinformatics 30, 360-368 (2014). 


\section{ONLINE METHODS}

Drug and immune cell gene expression data. Drug-induced transcriptional profile changes determined from human cancer cell lines were obtained from the Connectivity Map (CMap) database ${ }^{23}$. We processed and analyzed version 2, which included 6,100 experiments using 1,309 compounds. Preprocessing and normalization steps were performed, as described previously ${ }^{23}$. To make cross-platform comparisons compatible, we standardized gene identifiers from microarray-specific probe identifiers to NCBI GeneID identifiers, selecting the maximum across individual probe expression values. To create a single rank-ordered expression profile for each of the 1,309 compounds, we merged multiple experiments for the same compound into a single Prototype Ranked List (PRL) following the processing described previously ${ }^{28,29}$. The final data set included 13,071 differential gene expression values for each of the 1,309 compounds.

Immune cell gene expression data collected from steady-state profiling of 249 distinct cell types were obtained from the $\mathrm{ImmGen}^{24}$. Preprocessing and normalization were performed as described previously ${ }^{61}$. As cell profiles were collected at steady state, we selected 221 unique cell types and created 304 differential state signatures from the difference between two steady-state profiles (Supplementary Table 13). To make cross-species and cross-platform comparisons reasonable, we standardized gene identifiers from microarray-specific probe identifiers to NCBI GeneID identifiers, mapped mouse GeneID identifiers to their human ortholog, and selected the maximum across individual probe expression values. Finally, we converted differential state profiles to ranked lists ordered by differential expression values, creating a data set with 11,153 differential gene expression values for each of the 304 immunological state changes.

IP map construction. We constructed a matrix of predicted interactions between each of the 1,309 drugs and 304 immunological state changes using a rank-based, pattern-matching strategy described previously ${ }^{20}$. (All Input Data, and Supplementary Source Code have been deposited at Synapse hosted by Sage Bionetworks. https://www.synapse.org/\#!Synapse:syn4877787/files/) Briefly, for each trio of drug, cell and gene set size $(d, c, s)$, we calculated an immunemod score $(\operatorname{Im} S)$ based on the degree of overlap between drug and immune cell gene sets at the extremes of the two ranked signatures. To obtain a measure of significance for the immunemod score, we shuffled the genes in the drug rank signature and calculated a permuted immunemod score $\left(\operatorname{Im} S^{*}\right)$ for each drug, cell and gene set triplet $\left(\operatorname{ImS}^{*}\left(d_{i}, c_{j}, s_{k}\right)\right]$. We calculated the $P$ value for each $\operatorname{Im} S$ by counting the number of randomized scores $\operatorname{Im} S^{*}\left(d_{i}, c_{j}\right.$, $s_{k}$ ) that were greater than or equal to the absolute value of the actual scores $\operatorname{ImS}\left(d_{i}, c_{j}, s_{k}\right)$ and dividing by the number of permutations $\left(n_{\text {perms }}=1,000\right)$. This permutation strategy sets the lower bound for $P$-values at 0.001 , which yields a biased estimate for the number of false positives given the number of hypotheses under consideration. To provide accurate $P$-values at the lower range while containing the computational cost, we used the generalized Pareto distribution to model the $P$-value distribution and calculated improved estimates for low $P$-values (counts $<1 / 100$ ) based on the distribution of permuted immunemod scores ${ }^{62}$. We adjusted the $P$-values ${ }^{63}$ and selected an FDR of $5 \%$ as the cutoff for significance. To control for spurious interactions based on the size of the gene set used for matching, we varied the size of the matching set between 100 and 250 genes for each of the top and bottom extremes and recalculated all immunemod score, $P$-value pairs for every drug-cell interaction. The proportion of times each drug-cell interaction was significant among all sizes of gene sets provides a relative strength for any given interaction. A predicted interaction was considered to be strong and stable if it was significant for $85 \%$ or more of the set sizes.

Data analysis. To assess the similarity between expression profiles of immune cell subsets, we calculated the Jaccard distance among all pairs of extreme fold-change genes, and used an ANOVA to evaluate the differences between immune cell subsets. We investigated a series of diagnostic plots and did not find significant deviations that would violate the assumptions of normality or homoscedasticity.

To organize the drugs and immune cells in an unbiased manner, we applied hierarchical clustering to the full interaction matrix using the computed Pearson correlation coefficient as a distance metric between immune- mod scores and complete linkage clustering to agglomerate drugs or cells. We used the pvclust $\mathrm{R}$ package ${ }^{36}$ to compute a bootstrap analysis of the clusters and identified a significant cluster if the approximately unbiased probability was $>95 \%$.

To determine the enrichment of an anatomical therapeutic class category, we calculated the fold-change and $P$-value. Fold-change enrichment $(E)$ was calculated as a ratio of ratios $E=(a / b) /(c / d)$, where a is the number of drugs with a particular category (e.g., "L") in the cluster of interest, b is the number of drugs with that category in the overall data set, $\mathrm{c}$ is the total number of drugs in cluster and $\mathrm{d}$ is the total number of drugs overall. We used the hypergeometric distribution to calculate the $P$-value and assess the significance of each enrichment calculation.

To examine the association between chemical features (e.g., molecular targets and drug side effects) and number of immune cell interactions, we implemented a simple linear regression model. Chemical features followed a skewed distribution so we log-transformed the data, which adjusted the values so they followed a normal distribution. Based on diagnostic plots of the transformed data, we did not find deviations that violated the assumptions of normality and homoscedasticity that are central to regression models.

To test whether drug treatment with clioquinol or amantadine produced any difference in neutrophil cell frequencies in various tissue compartments, we used an ANOVA model to compare the treatment groups. Multiple group testing and $P$-values were evaluated using Tukey's honest significant difference. For all ANOVA tests, we generated a series of diagnostic plots to examine: (i) the residual errors for outliers, (ii) the QQ plots for normality, and (iii) the square root of the standardized residuals for heteroscedasticity. In all cases, we did not find significant deviations that would violate the assumptions used in the ANOVA model. For comparison, we evaluated the treatment and control groups directly via the Wilcoxon rank sum test and found the median differences between treatment (clioquinol, amantadine) and controls (PEG400, PBS) to follow the exact same pattern obtained using the ANOVA, with a similar maximum $P$-value for significant differences $(P \leq 0.01)$. To test whether guanfacine influence regulatory T-cell frequencies, we used a meta-analysis strategy ${ }^{64}$ to combine experimental conditions and groups, which allowed us to ascertain whether the overall differences from each independent experiment were robust and significant.

Electronic medical records. We pulled all patient entries from the Mount Sinai Electronic Medical Records that contained complete blood count information on neutrophils and monocytes (more than 2.3 million entries in total). To determine if either propofol or spironolactone were associated with a change in cell counts, we identified patient entries that had laboratory values measured within $30 \mathrm{~d}$ of drug administration versus patient entries that never received drug. We tested for group-level differences using the nonparametric Wilcoxon rank sum test. The findings were validated using the Electronic Medical Records of Columbia University Medical Center, where we employed the same criteria for patient selection and performed a Wilcoxon rank sum test for group differences.

Visualization. Circos plots created using the circlize R package (version 0.0.7 https://github.com/jokergoo/circlize). Network diagrams produced using Cytoscape $^{65}$ and SPADE trees generated with CytoSPADE ${ }^{66}$. All other plots created using the R statistical package.

Mice and drug treatment. 6- to 12 -week-old female $\mathrm{C} 57 \mathrm{Bl} / \mathrm{c}$ mice were obtained from Jackson Laboratories. Mice received intraperitoneal injections three times every $12 \mathrm{~h}$ with clioquinol, amantadine (both at $30 \mathrm{mg} / \mathrm{kg}$ per dose, Sigma Aldrich) or appropriate controls. Dosing level and frequency were chosen based on previous experiments using clioquinol in mice ${ }^{45,67}$ and the drug half-life (11-14 h). Clioquinol was dissolved in 8\% PEG400/PBS heated to $37^{\circ} \mathrm{C}$; amantadine was dissolved in PBS. Before injection, the solutions were shaken several times. Mice were euthanized for tissue collection between 2.5 and $3 \mathrm{~h}$ after the last treatment. Blood collection was obtained from the tail. For guanfacine treatment, mice received initial injection of $5 \mathrm{mg} / \mathrm{kg}$ of drug or vehicle control (PBS) on day 1 , followed by two (experiment 1 ) or six (experiments 2-5) intraperitoneal injections at $2 \mathrm{mg} / \mathrm{kg}$ every $12 \mathrm{~h}$ starting on day 2 . Mice were euthanized for tissue collection $12 \mathrm{~h}$ after the last treatment. 
All animal procedures were done according to protocols approved by the Mount Sinai School of Medicine Institutional Animal Care and Use Committee.

Flow cytometry. Peritoneal cavity cells were collected by washing with cold PBS containing 4\% FBS. Single-cell suspensions of bone marrow were obtained by flushing femurs, followed by filtration through a $100-\mu \mathrm{m}$ cell strainer (BD Biosciences). Red blood cells were lysed for $2 \mathrm{~min}$ at room temperature with $\mathrm{RBC}$ lysis buffer (eBioscience). Samples were stained with the following antibodies (all from eBioscience): allophycocyanin-eFluor780-conjugated CD45 (30-F11), peridinin chlorophyll protein-cyanine 5.5-conjugated CD11b (M1/70), phycoerythrin-conjugated Ly6G (RE6-8C5), phycoerythrin-conjugated CD3 (145-2C11), peridinin chlorophyll protein-cyanine 5.5-conjugated CD25 (PC61.5), fluorescein isothiocyanate-conjugated CD62L (MEL-14), allophycocyanin-conjugated FoxP3 (FJK-16s), eFluor450-conjugated CD4 (GK1.5), allophycocyanin-eFluor780-conjugated CD8a (53-6.7), and allophycocyaninconjugated CD44 (clone IM7, BD Pharmingen). DAPI was used to label dead cells. LSR Fortessa was used for sample acquisition and FlowJo software for data analysis.

RNA isolation and quantitative PCR. Total RNA was extracted from pieces of lung, liver, spleen and bone marrow cells using QIAzol Lysis Reagent (Qiagen) and glycogen blue (Ambion, Life Technologies) according to the manufacturer's instruction. For cDNA synthesis, $2 \mu \mathrm{g}$ total RNA was reversetranscribed for $1 \mathrm{~h}$ at $37^{\circ} \mathrm{C}$ with an RNA-to-cDNA kit (Applied Biosystems). For quantitative PCR, SYBR green qPCR master mix $2 \times$ (Fermentas, Thermo Scientific) and the following primers were used: mouse Actb forward, 5'-CTAAGGCCAACCGTGAAAAG- ${ }^{\prime}$, and reverse, $5^{\prime}$-ACCAGAGGCATACA GGGACA-3'; mouse Cxcl1 forward, 5'-GTGTTGCCCTCAGGGCC-3', and reverse, 5'-GCCTCGCGACCATTCTTG-3'; mouse Cxcl2 forward, 5'-ACGCCC CCAGGACCC- $3^{\prime}$, and reverse, $5^{\prime}$-CTTTTTGACCGCCCTTGAGA- $3^{\prime}$; mouse

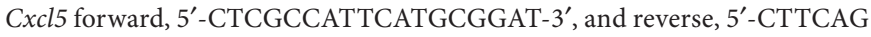
CTAGATGCTGCGGC-3'; mouse Cxcr2 forward, $5^{\prime}$-CTTTGCCCTGACC TTGCCT- ${ }^{\prime}$, and reverse, $5^{\prime}$-GCACAGGGTTGAGCCAAAA- ${ }^{\prime}$; mouse $C x c r 4$ forward, 5'-TGGCCTTCATCAGCCTGG-3' ${ }^{\prime}$, and reverse, 5' TGACTGTTGGTG-3'.

Mass cytometry (CyTOF) analysis of mouse spleen. To obtain single-cell suspensions, spleens were digested for $20 \mathrm{~min}$ at $37^{\circ} \mathrm{C}$ in HBSS containing $8 \%$ FBS and $0.2 \mathrm{mg} / \mathrm{ml}$ collagenase IV (Sigma Aldrich). After filtration through a $100-\mu \mathrm{m}$ cell strainer, red blood cells were lysed for $2 \mathrm{~min}$ at RT with RBC lysis buffer. Cells $\left(5 \times 10^{6}\right.$ per sample) were stained for the following surface markers: 141Pr-Ly6G, 153Eu-PDCA1, 162Dy-Ly6C, 166Er-CD138 (all prepared in-house) and 142Nd-CD11c, 147Sm-CD45, 148Nd-CD11b, 149Sm-CD19, 151Eu-CD25，152Sm-CD3e, 156Gd-Thy1.2，160Gd-CD62L，168Er-CD8, 169Tm-TCRb, 170Er-NK1.1, 171Yb-CD44, 172Yb-CD4, 174Yb-MHCII and $176 \mathrm{Yb}-\mathrm{B} 220$ (all from DVS Sciences). Cisplatin was added for the final $5 \mathrm{~min}$ to label dead cells and samples were fixed using Fix and Perm buffer (DVS Sciences). Immediately before injection, EQ Four Element Calibration Beads were added and samples were run on CyTOF 2 mass cytometer (DVS Sciences) in three 10-min acquisitions rounds. Data were normalized to EQ Beads and files concatenated using DVS software.

61. Painter, M.W. et al. Transcriptomes of the $B$ and $T$ lineages compared by multiplatform microarray profiling. J. Immunol. 186, 3047-3057 (2011).

62. Knijnenburg, T., Wessels, L., Reinders, M. \& Shmulevich, I. Fewer permutations, more accurate P-values. Bioinformatics 25, i161 (2009).

63. Benjamini, Y. \& Hochberg, Y. Controlling the false discovery rate-a practical and powerful approach to multiple testing. J. R. Stat. Soc., B 57, 289-300 (1995).

64. Morgan, A.A., Khatri, P., Jones, R.H., Sarwal, M.M. \& Butte, A.J. Comparison of multiplex meta analysis techniques for understanding the acute rejection of solid organ transplants. BMC Bioinformatics 11 (suppl. 9), S6 (2010).

65. Shannon, P. et al. Cytoscape: a software environment for integrated models of biomolecular interaction networks. Genome Res. 13, 2498-2504 (2003).

66. Linderman, M.D. et al. CytoSPADE: high-performance analysis and visualization of high-dimensional cytometry data. Bioinformatics 28, 2400-2401 (2012).

67. Choi, B.Y. et al. Copper/zinc chelation by clioquinol reduces spinal cord white matter damage and behavioral deficits in a murine MOG-induced multiple sclerosis model. Neurobiol. Dis. 54, 382-391 (2013). 